\title{
Binding specificity and in vivo targets of the EH domain, a nove protein-protein interaction module
}

\author{
Anna Elisabetta Sal cini, ${ }^{1}$ Stefano Confal onieri, ${ }^{1}$ Margherita Doria, ${ }^{1}$ Elisa Santolini, ${ }^{1}$ Elena Tassi, ${ }^{1}$ \\ Olga Minenkova, ${ }^{2}$ Gianni Cesareni, ${ }^{2}$ Pier Giuseppe Pelicci, ${ }^{1,3}$ and Pier Paolo Di Fiore ${ }^{1,4,5}$ \\ ${ }^{1}$ Department of Experimental Oncology, European Institute of Oncology, Milan 20140, Italy; ${ }^{2}$ Dipartimento di Biologia, \\ University of Rome “Tor Vergata," Rome 00100, Italy; ${ }^{3}$ sstituto di Patologia Speciale M edica, University of Parma, Parma \\ 43100, Italy; ${ }^{4}$ Instituto di Microbiologia, University of Bari, Bari 70100, Italy
}

\begin{abstract}
EH is a recently identified protein-protein interaction domain found in the signal transducers Eps15 and Eps15R and several other proteins of yeast nematode. We show that EH domains from Eps15 and Eps15R bind in vitro to peptides containing an asparagine-proline-phenylal anine (NPF) motif. Direct screening of expression libraries with EH domains yielded a number of putative EH interactors, all of which possessed NPF motifs that were shown to be responsible for the interaction. Among these interactors were the human homolog of NU MB, a developmentally reguated gene of D rosophila, and RAB, the cellular cofactor of the HIV REV protein. We demonstrated coimmunoprecipitation of Eps15 with NUMB and RAB. Finally, in vitro binding of NPF-containing peptides to cellular proteins and EST database screening establ ished the existence of a family of EH-containing proteins in mammals. Based on the characteristics of EH-containing and EH-binding proteins, we propose that EH domains are involved in processes connected with the transport and sorting of molecules within the cell.
\end{abstract}

[Key Words: EH domain; protein interaction; Eps15; internal ization; trafficking]

Received May 21, 1997; revised version accepted July 2, 1997.

Many cellular functions, including proliferation, differentiation, cytoskeleton organization, and apoptosis, are regulated through a complex intracellular network of signal transducers. Specialized protein domains, such as $\mathrm{SH} 2, \mathrm{SH} 3, \mathrm{PTB}$, or WW, mediate this vast array of interactions by binding to specific sequences located in target proteins (for review, see M usacchio et al. 1994; Cohen et al. 1995; van der Geer and Pawson 1995 and references therein). Within individual groups, binding domains have diverged to recognize specific consensus sequences in which some positions are obligatory occupied by given amino acids and other can vary. For example the SH2 and PTB domains both recognize phosphotyrosinecontaining motifs (Songyang et al . 1993, 1994; Blaikie et al. 1994; Kavanaugh and Williams 1994; Zhou et al. 1995). Specificity appears to be determined by variations in amino acids distal or proximal to the phosphotyrosine residue for $\mathrm{SH} 2$ and $\mathrm{PTB}$ domains, respectively (for review, see Cohen et al. 1995; van der Geer and Pawson 1995). SH3 domains, on the other hand, interact with prolinerich peptides in target proteins (Cichetti et al. 1992; Gout et al. 1993; Ren et al. 1993; Yu et al. 1994). Screening of biased random libraries all owed the identi-

${ }^{5}$ Corresponding author.

E-MAIL pdifiore@ieo.cilea.it; FAX 39-2-57489851. fication of two classes of $\mathrm{SH} 3$ ligands, conforming to the general structure RXLPPZP (class 1 ) and XPPLPXR (class 2 ), whereas $X$ is any amino acid other than $C$ and $Z$ is either L or R (Feng et al. 1994; Schlessinger 1994).

$\mathrm{SH} 2$ and $\mathrm{SH} 3$ domains contribute to the propagation of signals by affecting the subcellular localization of the domain-containing proteins or their targets, or by recruting these proteins to specific enzymes (for review, see Cohen et al. 1995). Recent evidence, however, suggests that these domains might not serve solely as passive binding interfaces. In the case of $\mathrm{SH} 3 \mathrm{~s}$, in particular, it has been shown that binding to the dynamin GTPase and to phosphatidylinositol-3' kinase (PI-3K) activates these enzymes in vitro (Gout et al. 1993; Herskovits et al. 1993; Pleiman et al. 1994).

Finally, a phylogenetic hierarchy of interactions might also exist, as witnessed by the presence of some binding domains, such as $\mathrm{SH} 3$ and WW in lower eukaryotes like yeast, and the appearance of others, such as $\mathrm{SH} 2$ and PTB, concomitantly with the emergence of tyrosine kinase-mediated signaling in multicellular eukaryotes (for review, see Cohen et al. 1995).

We have recently described a novel protein-protein interaction domain, called EH (for Eps15 homology), present in three copies in the amino terminus of the tyrosine kinase substrates Eps15 and Eps15R and shared with 
other proteins of yeast and nematode (Wong et al . 1995). Except for its protein-binding ability, no other clues to the function(s) of the $\mathrm{EH}$ domain are available, as $\mathrm{EH}$ containing proteins appear to serve different roles in celI ular homeostasis. One EH-containing protein, End3p, is involved in the internalization of the yeast $\alpha$-mating factor (Benedetti et al. 1994); another, Panlp, is requi red for normal organization of the actin cytoskeleton in yeast (Tang and Cai 1996). Other EH-containing proteins still have unknown functions. However, mutagenesis studies in END3 suggested that the presence of its EH domain is necessary for function (Benedetti et al. 1994). Understanding of the fuctions of $\mathrm{EH}$ will ultimately rely on the identification of its binding specificity and of the intracellular targets recruited in a hypothetical EH-based network. Studies described in this paper were undertaken to shed light on these issues.

\section{Results}

EH domains from Eps15 and Eps15R bind to NPF-containing peptides

In their amino termini, Eps15 and Eps15R contain three copies of a novel protein-protein interaction domain, which we named EH (Fig. 1A). To understand the binding specificity of EH domains, we engineered glutathione Stransferase (GST) fusion proteins containing the three EH domains of Eps15 (GST-EH) or Eps15R (GST-EHR). These fusion proteins were employed to screen a random phagedisplayed peptide library, and the selected phages were sequenced in the region corresponding to the random inserts. In Figure 1B, the conceptually translated peptides are displayed. Of 48 selected peptides, 46 contained the motif NPF (asparagine-proline-phenylalanine). Two additional phages containing either NHF (asparagine-histidine-phenylalanine) or HPF (histidine-proline-phenylal anine) were also selected by GST-EHR (Fig. 1B). Position +1 (with respect to NPF) exhibited a strong preference for basic and basic/hydrophobic residues in peptides selected by GST EHR and GST-EH, respectively. Of note, negatively charged residues were never present in this position. Positions -1 and -2 displayed a weaker preference for serine or threonine (Fig. 1B).

Cellular proteins binding to $\mathrm{EH}$ in vitro invariably contain NPF motifs

We have shown previously that the GST-EH fusion pro-
Figure 1. EH-mediated binding to peptides and proteins in vitro and in vivo. (A) Schematic of the Eps15 and Eps15R proteins with their $\mathrm{EH}$ domains. Amino acids positions are indicated. (B) Predicted amino acid sequence of peptides selected by screening of a random phage-displayed peptide library with GST-EH and GSTEHR, from Eps15 and Eps15R, respectively. NPFs are in boldface type. (C) N PF-containing motifs present in the cDNAs identified by screening of a human fibroblast expression Iibrary using GST -EH as a probe. Underlined peptides were used for the in vitro binding experiments described in Figs. 4, 6, and 8.
A

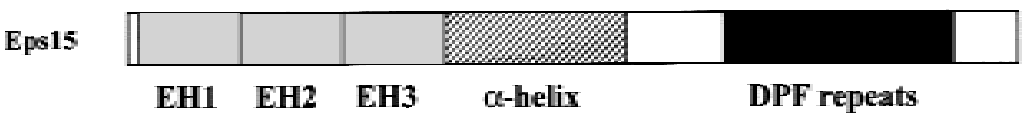

Epsis R

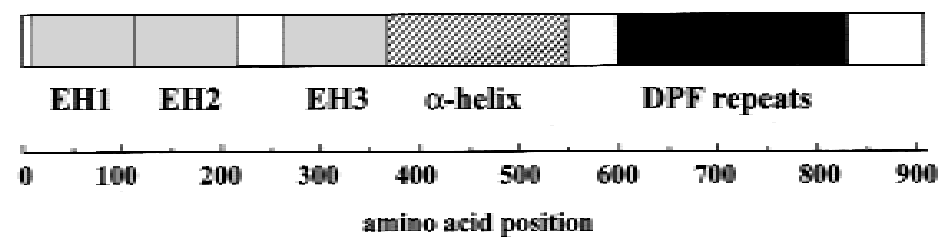

B

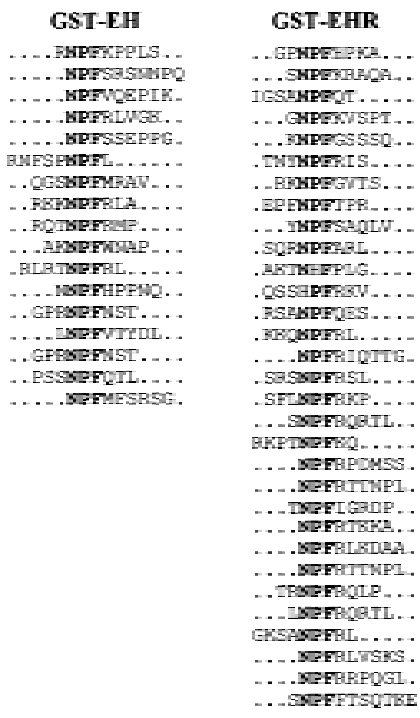

C

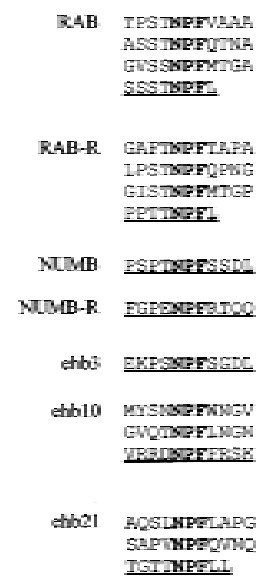

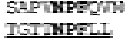


tein is able to specifically bind to a number of cellular proteins, in Far Western assays (Wong et al. 1995). To clone the cDNAs encoding these proteins, we employed the GST-EH fusion protein to screen a prokaryotic expression library from M 426 human fibroblasts. We identified several positive plaques that specifically reacted with GST-EH but not with control GST. By cross-hybridization experiments, the positive phages could be assigned to seven groups, corresponding to distinct CDN As (not shown).

$\mathrm{N}$ ucleotide sequence of the longest phage inserts of these cDNAs revealed that they represented partial CDN A s of the human homolog of NU MB, a devel opmentally regulated gene of Drosophila (Uemura et al. 1989); a NU MB-related gene, which we named NU MB-R; RAB, coding the cellular cofactor of the HIV REV protein (Bogerd et al. 1995; Fritz et al. 1995); and a RAB-related gene, which we named RAB-R (see next section). We al so identified three novel genes that we named ehb3, ehb10, and ehb21 (for $\mathrm{EH}$ binding, followed by the original plaque identifier; datā not shown).

There were no homologies among the seven partial cDN As (apart from those between NUM B and NUMB-R, and $R A B$, and RAB-R, respectively), except for the presence of N PF motifs, which frequently are present in multiple copies (Fig. 1C). Alignment of all N PF-containing stretches reveal ed preference for hydrophobic resi dues at position +1 (relative to NPF) and for S/T at positions -1 and -2 (Fig. 1C).

Cloning of CDNAs containing entire ORFs for human NUMB, NUMB-R, RAB, and RAB-R and characterization of their predicted products

The cDN As obtained from the screening of the prokaryotic expression library represented partial cDNAs that did not contain entire open reading frames (ORFs) (data not shown). We therefore screened a human fibroblast cDNA library to obtain the entire ORFs of human RAB, RAV-R, NU MB, and NU MB-R. Several CDNAs were isolated, and the longest ones, representative of each gene, were sequenced. A schematic of the CDN As containing the entire ORFs of human NUMB and NUMB-R and of human $R A B$ and RAB $R$ is presented in Figures $2 A$ and $3 A$, respectively, and details are given in the figure legends. The sequence of human RAB corresponded to that al ready reported by Bogerd et al. (1995) and Fritz et al. (1995). Conceptual translation of the cDN As al lowed for comparison of human NUMB and NUMB-R (Fig. 2B) and RAB and RAB-R (Fig. 3B).

The ORFs of human NUMB and NUMB-R have the capacity of encoding peptides of 603 and 609 amino acids, respectively, with a predicted molecular mass of 66 and $65 \mathrm{kD}$. The two predicted proteins display an overal I relatedness of $74 \%$ with $57 \%$ identity (Fig. 2B). As already reported for murine and Drosophila (Verdi et al. 1996; Zhong et al. 1996), both human NUMB and NUMB-R contained a phosphotyrosine interaction domain (PID/PTB; van der Geer and Pawson 1995) in their amino terminus (Fig. 2B), and putative SH3-binding sites
A

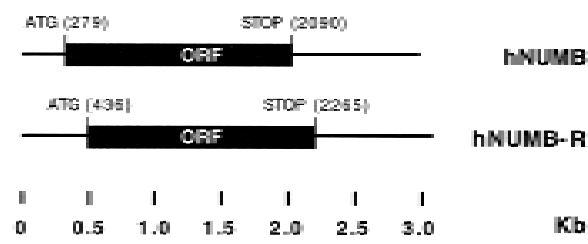

B

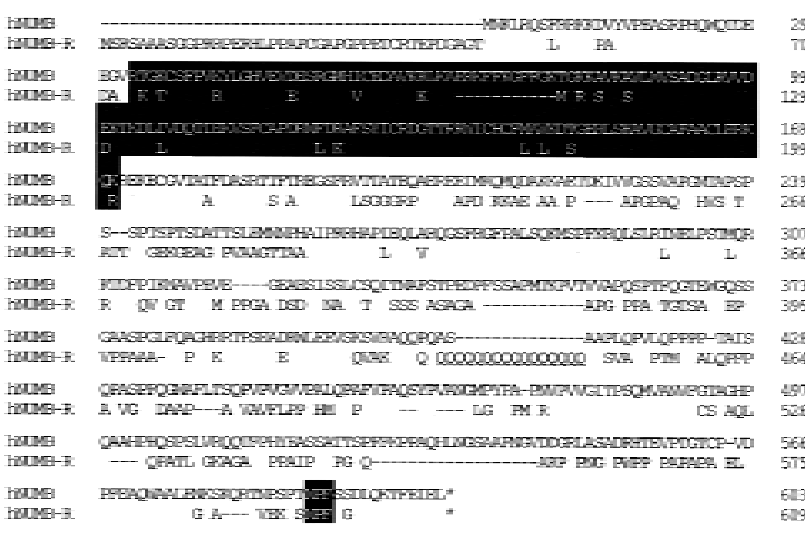

Figure 2. Human NUMB and NUMB-R CDNAs and proteins. (A) The structures of the human NUMB (hNUMB) and NUMB-R (hN UMB-R) CDNAs are depicted. The ORFs are labeled. Positions are indicated in $\mathrm{kb}$. The nucleotide positions of initiator and terminator codons are also indicated. Canonical polyadenylation sites (AATAAA) are at positions 2649, 2823, 2958, and 3025, for hNU MB and hNU MB-R, respectively (not shown). (B) Predicted protein sequences and alignment of human NUMB and NUMB-R. In the hNUMB-R sequence, only nonidentical amino acids are reported, except for the N PF motifs. Dashes indicate gaps introduced to maximize the alignment. Accepted conservations, employed to calculate relatedness, are $D, E, N, Q ; L, I, V, M ; K, R, H ; F, Y, W$; and $A, G, P, S$, $T$. The PT B domains and the N PF motifs are indicated in reverse print.

in their carboxyl terminus. In addition, they both contained a single NPF motif, located a few amino acids before the end of the proteins (Fig. 2B).

The ORFs of human RAB and RAB-R have the capacity of encoding peptides of 562 and 481 amino acids, respectively, with a predicted molecular mass of 58 and $49 \mathrm{kD}$. The two predicted proteins display an overall relatedness of $71 \%$ with $46 \%$ identity (Fig. 3B). Conserved features between RAB and RAB-R include a zinc finger region, in the amino terminus of the proteins, and several FG (phenylalanine-glycine) motifs, characteristic of nucleoporin-like proteins (Bogerd et al. 1995, Fritz et al. 1995). In addition, they both contained four NPF motifs, located in the carboxy-terminal half of the molecule (Fig. 3B). It has not been established whether all of the N PF motifs are endowed with EH-binding ability, and if so, whether they bind to different $\mathrm{EH}$-containing proteins. 
A

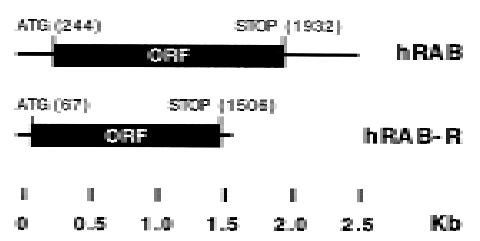

B

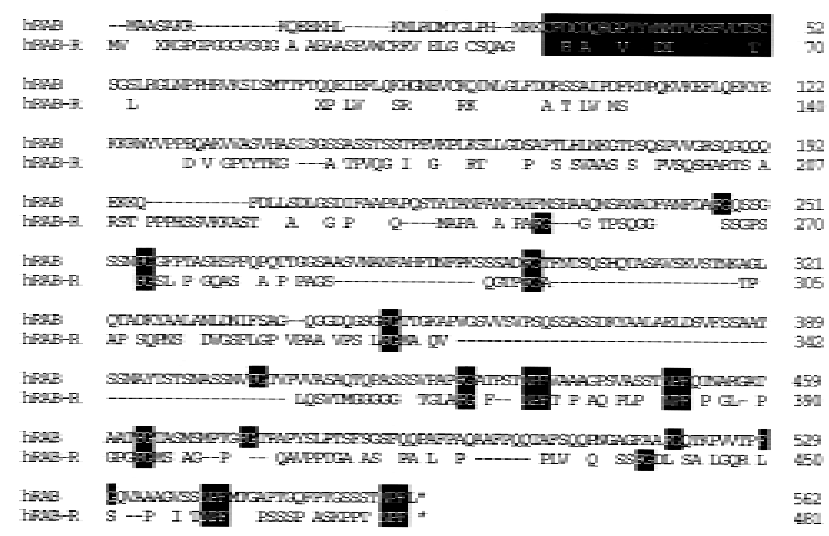

Figure 3. Human RAB and RAB-R CDNAs and proteins. (A) The structures of the human RAB (hRAB) and RAB-R (hRAB-R) CDN As are depicted. The ORFs are labeled. Positions are indicated in $\mathrm{kb}$. The nucleotide positions of initatior and terminator codons are also indicated. Canonical polyadenylation sites (AATAAA) are at positions 2499, 2542, and 2556 of the RAB sequence; no polyadenylation site was found in the isolated hRAB-R CDNA (not shown). (B) Predicted protein sequences and alignment of human RAB and RAB-R. The sequence of hRAB is identical to that reported by Bogerd et al. (1995) and Fritz et al. (1995). In the hRAB-R sequence, only nonidentical amino acids are reported, except for the FG and NPF motifs. Dashes indicate gaps introduced to maximize the alignment. Accepted conservation, employed to calculate relatedness, are $D, E, N, Q ; L, I, V, M ; K, R, H ; F, Y, W$; and $A, G, P, S, T$. The FG, zinc-finger, and N PF motifs are indicated in reverse print.

Binding of various cellular proteins to $\mathrm{EH}$ domains in vitro and in vivo

To characterize the binding of the $\mathrm{EH}$ interactors to Eps15, we engineered GST fusi on proteins encompassing fragments derived from the seven identified $\mathrm{EH}$-binding proteins. In all cases, the original partial CDNAs were used (see M aterials and M ethods), as they should contain all of the determinants necessary and sufficient for $\mathrm{EH}$ binding. As shown in Figure 4A, all of the GST fusion proteins were able to specifically recover native Eps15 from cell lysates in vitro binding experiments.

To address the issue of whether the NPF-containing peptides, present in the EH-binding proteins, were actually responsible for binding to Eps15, we engi neered GST fusi on proteins encompassing short N PF-containing peptides from the proteins of interest. The peptides engi- neered as GST fusions are underlined in Figure 1C. Also in this case (Fig. 4B), we evidenced specific binding of the GST peptide fusions to native Eps15 from total cellular lysates. Although the participation of other regions of the EH-interacting proteins to the binding cannot be excluded, the sum of the above results indicates that N PFcontaining peptides are sufficient for binding to Eps15.

We then tested whether Eps15 can physically interact with some of the EH-binding proteins in vivo. To this end, we utilized the GST-NUMB and GST-RAB fusion proteins, described in Figure $4 A$, as immunogens to generate polyconal sera. The anti-RAB and anti-N UMB sera recognized specifically proteins of $\sim 60 \mathrm{kD}$ and a doublet of $70-72 \mathrm{kD}$, respectively, in several human and mouse cell lines (data not shown). The two sera were then used to coimmunopreci pitate Eps15 from lysates of N IH-3T 3 cells (Fig. 5A) or of U2OS cells (not shown). As shown in Figure 5A, Eps15 was recovered in immunoprecipitates obtai ned with anti-N UMB or anti-RAB sera but not with control sera.

The amount of Eps15 coimmunoprecipitated by NUMB was higher than that coimmunoprecipitated by RAB. Although this might be attributable to a lower affinity of the RAB/Eps15 interaction, we favor the possibility that it simply reflects less efficient immunoprecipitation of RAB by the anti-RAB antibody. In another set of experiments, we engineered eukaryotic expression vectors encoding for either NUMB or RAB fused to a
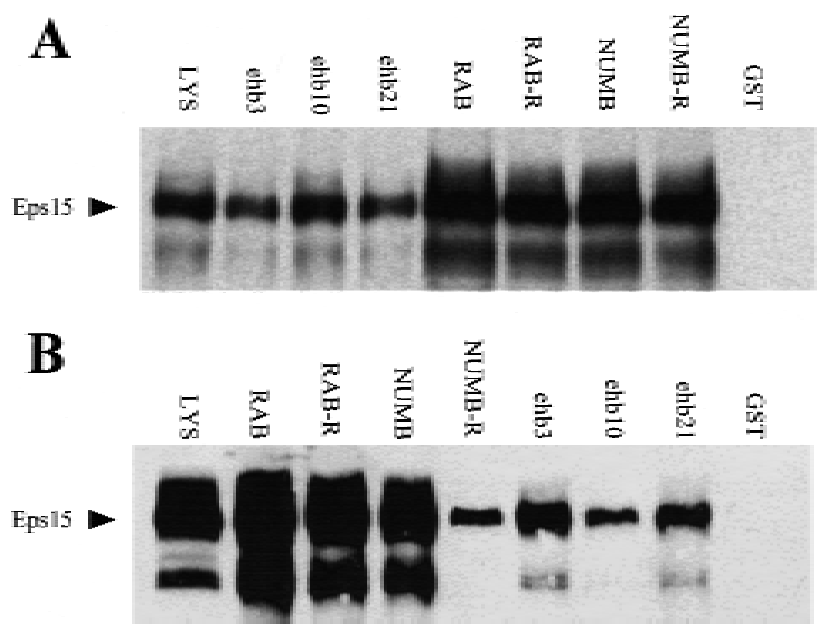

Figure 4. In vitro binding of N PF-containing proteins and peptides to Eps15. (A) In vitro binding of Eps15 to Gst fusions of EH-binding proteins. Total cellular Iysates from N IH-3T 3 cells (1 mg/lane) were incubated with the indicated GST fusion proteins $(10 \mu \mathrm{g})$ for $1 \mathrm{hr}$ at $4^{\circ} \mathrm{C}$. Specifically bound Eps 15 was detected by immunoblot with an anti-Eps15 antibody. (B) Binding of Eps15 to GST fusions encompassing NPF-containing peptides. GST fusion proteins were engineered to encompass the N PF-containing peptides underlined in Fig. 1C and are indicated with the names of the proteins from which the peptides were derived. In vitro binding experiments were performed as described in A. Lanes marked LYS in A and B were loaded with 50 $\mu \mathrm{g}$ of total cellular proteins to serve as a reference for the position of Eps15. The position of Eps15 is also indicated. 


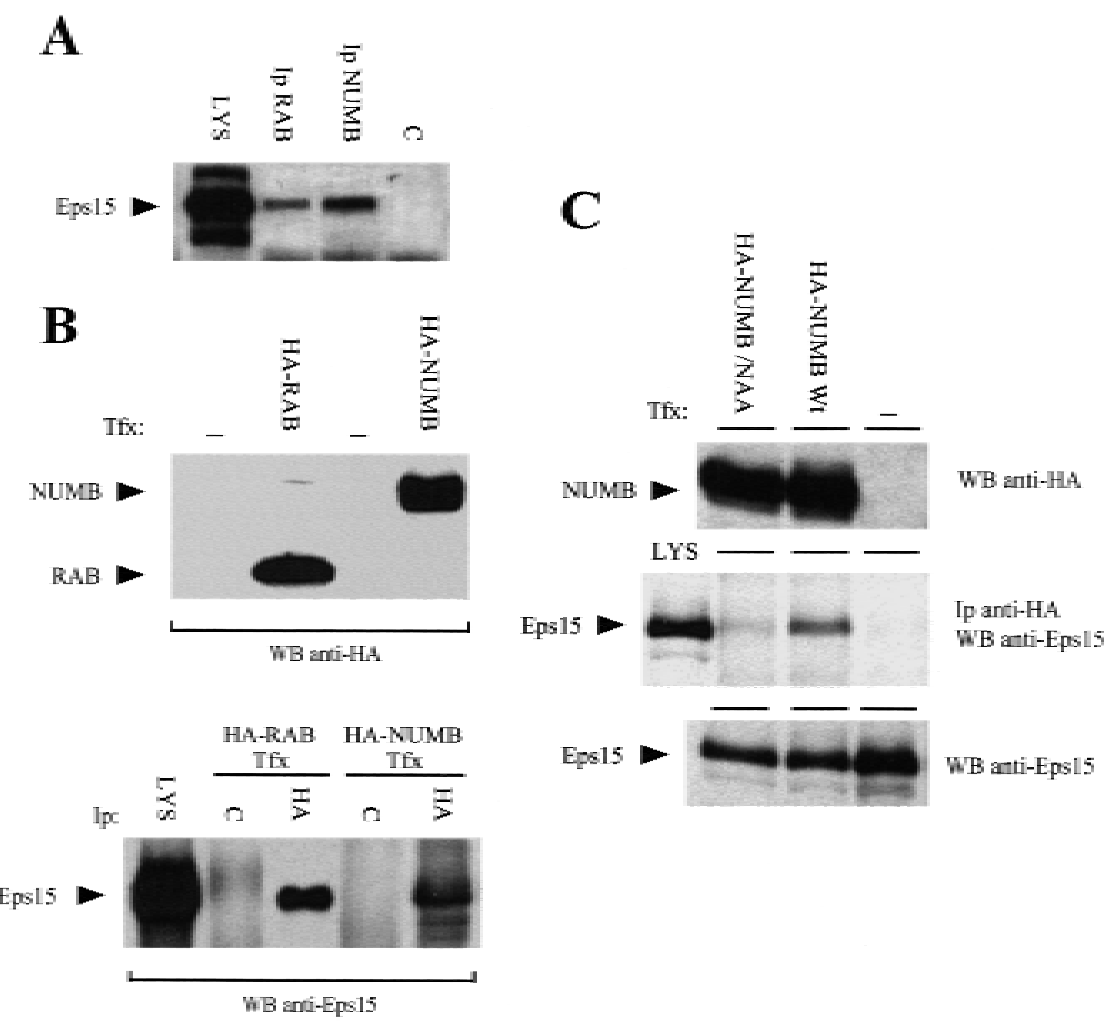

Figure 5. In vivo binding of NPF-containing proteins to Eps15. (A) Coimmunoprecipitation of Eps15 with endogenously expressed NUMB and RAB. Total cellular proteins from NIH-3T3 cells were obtained under mild lysis conditions to preserve protein-protein interactions, and 5 mg of protein was immunoprecipitated with an anti-RAB (Ip RAB), an anti-N UMB (Ip NUMB), or a control serum (C). After SDS-PAGE, coimmunoprecipitating Eps 15 was detected by immunoblot with an antiEps15 antibody. (B) Eps15 coimmunoprecipitation with HA-tagged RAB and NUMB proteins. (Top) C 33A cells were transfected with expression vectors engineered to express $H A$-tagged RAB or NUMB proteins (HA-RAB or HA-NUMB lanes, respectively) or mock transfected (lanes). Total cellular lysates $(100 \mu \mathrm{g})$ were immunoblotted with an anti-HA antibody. (Bottom) Five milligrams of total cellular proteins from HA-RAB or HA-NUMB transfectants (HA-RAB TfX and HANUMB Tfx, respectively) obtained as in A were immunoprecipitated with the antiHA antibody (HA lanes) or with a control serum (C Ianes) and detected by immunoblot with an anti-eps15 antibody. (C) Eps15 coimmunoprecipitation with HA-tagged NUMB and NUMB mutant. C33A cells

were transfected with expression vectors encoding HA-tagged NUMB or an HA-tagged NUMB mutant in which the N PF motif was mutagenized to N AA (lanes HA-NUMB and HA-N U MB/NAA, respectively), or mock transfected (-lane). (Top) Total cellular lysates $(100 \mu \mathrm{g})$ were immunoblotted with an anti-HA antibody; (middle) $5 \mathrm{mg}$ of total cellular proteins obtained as in A was immunoprecipitated with an anti-HA antibody and detected by immunoblot with an anti-Eps15 serum; (bottom) total cellular lysates (100 $\mu \mathrm{g}$ ) were immunoblotted with an anti-Eps15 antibody. Lanes marked LYS in A-C were loaded with $50 \mu \mathrm{g}$ of total cellular proteins to serve as a reference for the position of Eps15. The positions of Eps15, NUMB, and RAB are also indicated.

hemagglutinin (HA) epitope, at their amino termini. C33A cells were then transiently transfected with vectors coding HA-NUMB or HA-RAB. As shown in Figure $5 B$ (top) HA-NUMB and HA-RAB were expressed at similar levels. Under these conditions, Eps15 was readily and comparably recoverable by immunoprecipitation with an anti-HA antibody but not by control antibodies, in both HA-NUMB or HA-RAB transfectants (Fig. 5B, bottom). The sum of the above results strongly argues that Eps15 can interact with NUMB and RAB in vivo.

To prove that the in vivo interaction of Eps15 with a representative $\mathrm{EH}$-binding protein protein was attributable to a N PF-medi ated interaction, we engineered a HAtagged mutant of NUMB (HA-NUMB/NAA) in which its unique N PF motif was mutageneized to N AA (asparagine-alanine-alanine). U pon transfection in C 33 A cells, both the HA-NUMB/NAA and the wild-type HANUMB mutants were expressed at compatable levels (Fig. 5C, top). In HA-NUMB/NAA transfectants, however, little if any Eps15 was visible in anti-HA immunoprecipitates, whereas it was readily detectable in similar immunoprecipitates obtained from wild-type HAN U M B transfectants (Fig. 5C, middle). These differences were not attributable to the levels of expression of en- dogenous Eps15, which appeared comparable in all transfectants (Fig. 5C, bottom). Thus, the in vivo binding of Eps15 to N U M B is most likely mediated by an EH-N PF interaction.

\section{Characterization of the NPF-EH interaction}

To assess the relevance of the N PF motif and of the surrounding positions for binding to $\mathrm{EH}$, we selected the peptide SSSTNPFL, which is present in RAB and strongly binds to Eps 15 and mutated individual amino acid positions in a GST fusion protein background. The various GST fusion proteins depicted in Figure 6 (left panels) were then tested for their ability to recover native Eps15 from cellular lysates in in vitro binding assays.

Mutations in the NPF motif to NGF, DPF, and NPY abol ish binding completely (Fig. 6A, right). We then performed alanine scanning of the positions surrounding the N PF motif. This unconvered a hierarchy of contribution to binding, as $A(-1), A(-2)$, and $A(+1)$ mutants, containing mutations to al anine at positions $-1,-2$, and +2 respective to the NPF motif, displayed binding reduced by $95 \%, 60 \%$, and 55\%, respectively (Fig. 6B, 
Figure 6. Requirement for the N PF motif and surrounding positions for binding to Eps15. (A) Binding to Eps15 of mutant peptides containing mutations in the N PF sequence. Peptides engineered in GST fusion proteins are indicated on the left. GSTNPF corresponds to the sequence of a NPF-containing peptide derived from the sequence of RAB (underlined in Fig. 1C). Mutant peptides (GST-NGF, GST-DPF, GST-N PY) are al so indicated. The in vitro bindings to Eps15, obtained as described in Fig. 4, are shown on the right. The lane marked RAB represents an in vitro binding obtained with the GST-RAB protein (Fig. $4 \mathrm{~A}$ ) to serve as a positive control. (B) Alanine scanning. Positions surrounding the NPF motif in the RAB peptide were alanine scanned as indicated on the left. The in vitro binding to Eps15, obtained as described in Fig. 4, are shown on the right. Lanes marked LYS were loaded with $50 \mu \mathrm{g}$ of total cellular proteins to serve as a reference for the position of Eps15 (also indicated). Amino acids corresponding to mutagenized codons are shown in boldface type.

right). Mutations of residues -3 and -4 to alanine did not appreciably affect binding (Fig. 6B, right). Finally a GST peptide bearing five simultaneous mutations to alanine (A5 mutant), leaving the N PF motif intact, displayed little if any detectable binding (Fig. 6B, right). These results indicate that an intact NPF is necessary but not sufficient for binding and that optimal binding is conditioned by the presence of certain amino acids in the surrounding positions. Whether this is attributable to impact on conformation of directly on binding remains to be established.

A single $\mathrm{EH}$ is necessary and sufficient for interaction with an EH-binding protein

$M$ any EH-containing proteins display repeated copies of the binding module (Wong et al. 1995); this observation raises the question as to whether a single $\mathrm{EH}$ is endowed with binding ability or whether multiple copies are required. Our screenings of phage-displayed and bacterial expression libraries were performed with GST fusion proteins containing the three $\mathrm{EH}$ domains of Eps15 and Eps15R, to maximize the chance of detecting proteinprotein interactions. To design a strategy to engineer GST fusions containing single EH domains, we had to take into account the fact that the EH domains of Eps15 are contiguous. Thus, definition of the exact boundaries and of the structural consequences of extrapolating sequences from their natural context was difficult to predict.

We thought, therefore, to take advantage of secondary structure predictions of the amino terminus of Eps15. As shown in Figure 7A, a Chous-Fasman-Rose algorithm predicted that the three EH domains of Eps15 are flanked by regions with high propensity for turns, possibly underlying the requirement for domain exposure, to achieve binding. Thus, we engineered a GST fusion protein containing residues 106-216 of Eps15, which en- compass the second EH flanked by the predicted amino and carboxy-terminal turn (M2 protein, Fig. 7B). This protein displayed strong binding to RAB, obtained by in vitro transcription/translation (Fig. 7C). In addition, binding of GST -M 2 to RA B was quantitative and of comparable intensity to that obtained with GST-EH (Fig. 7C), thus establishing that an individual EH domain is endowed with binding ability.

\section{A family of EH-containing proteins in mammals}

Eps15 and Eps15R are the only EH-containing proteins known in mammals. The identification of peptide sequences that bind to $\mathrm{EH}$ allowed for the identification of putative EH-containing proteins in mammalian cells. GST fusion proteins, encompassing N PF-contai ning peptides from NUMB, RAB, and RAB-R were challenged with ${ }^{35} \mathrm{~S}$-labeled lysates from N IH -3T 3 cells, and several cellular proteins were specifically recovered (Fig. 8A). In the case of the RAB peptide, mutations of NPF to DPF, NGF, or NPY totally abolished recognition, arguing strongly that the identified proteins represent EH-containing species (Fig. 8A). In addition, individual NPFcontaining peptides were able to bind to both common and different proteins, thus indi cating a certain degree of specificity for EH-mediated interactions.

The existence of a family of $\mathrm{EH}$-containing protein in mammal s was confirmed by screening the Expressed Se quence Tags Database (dbEST) with the EH domains of Eps15 and Eps15R. Four novel mammalian sequences, two from Homo sapiens and two from Mus musculus, were identified that contained EH domains (Fig. 8B).

\section{Discussion}

Data presented in this study establish the existence of an expanding family of EH-containing proteins and of a network of EH-mediated interactions. Furthermore, they 


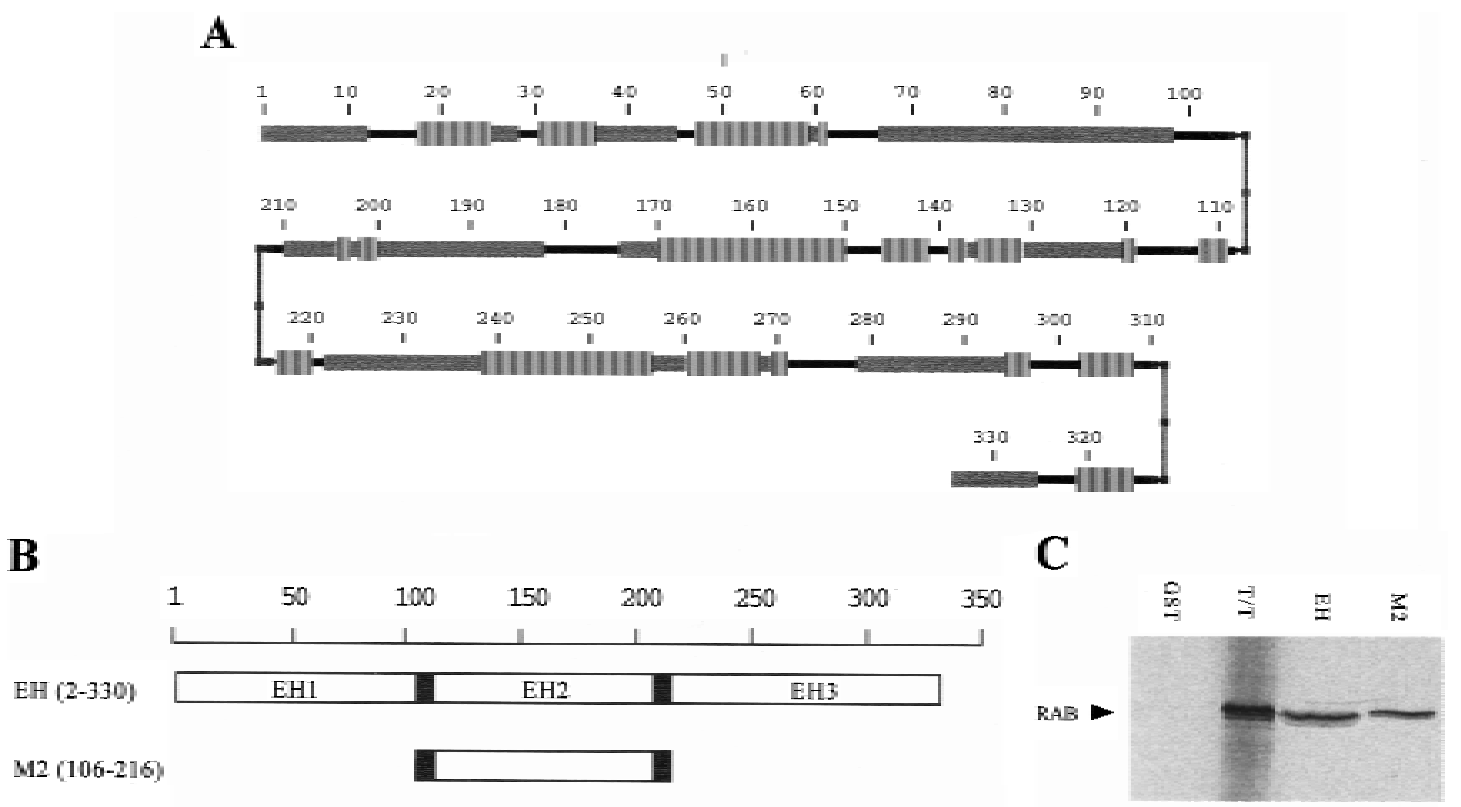

Figure 7. M apping of the minimal region of eps 15 required for binding to N PF-containing proteins. (A) Secondary structure prediction of the amino-terminal region of Eps15 (containing the three EH domains) by a Chou-Fasman-Rose algorithm. (Vertical lines) $\alpha$-Helices; (thick shaded bars) $\beta$-sheets; (thinner solid bars) coils; (small solid boxes) turn. Amino acid positions are also indicated. (B) Schematic representation of the Eps15 amino-terminal domain and of the GST fusion proteins engineered, with predicted turns indicated by solid boxes. The indicated fragments of Eps15 were engineered into GST fusion proteins and used for in vitro binding experiments. The EH construct contains all three $\mathrm{EH}$ domains. The $\mathrm{M} 2$ construct contains the region encompassing the second $\mathrm{EH}$ domain flanked by the natural regions pedicting the turns shown in A. Amino acid positions are also indicated in parentheses. (C) In vitro bindings. The GST fusions shown in B were used to bind the ${ }^{35} \mathrm{~S}$-labeled RAB protein, obtained by in vitro transcription/ translation of the RAB CDNA. Detection was by autoradiography. The lane marked T/T was loaded with the primary product of the in vitro transcription/translation to serve as a reference. The positions of RAB are also indicated.

define the molecular basis of EH-mediated interactions of two members of this family, Eps15 and Eps15R, and their binding to NPF-containing peptides. We do not know whether other EH domains will display similar binding preferences. By analogy to other binding modules, it is conceivable that critical binding residues will be invariant or conserved. However, a certain degree of variation is expected, as EH domains are only $~ 40 \%$ homologous. SH3 domains, by analogy, are 30\% homologous, and their binding preferences vary (Sparks et al. 1996).

With regard to Eps15, several proteins are known to date that interact with it, including $\mathrm{Crk}$, through its $\mathrm{SH} 3$ domain (Schumacher et al . 1995), the coated-pits adapter AP-2 (Benmerah et al. 1995, 1996; Iannolo et al. 1997), and the EH-binding proteins described in this paper. Thus, Eps15 is a multibinding protein that might function in the assembling of macromolecular complexes. We do not know whether all of the Eps 15 targets interact simultaneously with it or whether a hierarchy of interaction exists in vivo.

A wealth of evidence now supports the notion that $\mathrm{EH}$ domains are involved in receptor-mediated internalization. The first indication derived from the identification of EH domains in End3p, a yeast protein essential for internalization of the $\alpha$-mating factor and of its receptor
Ste2p (Burkholder and Hartwell 1985; N akayama et al. 1985; Benedetti et al. 1994; Wong et al. 1995). A nother $\mathrm{EH}$-containing yeast protein, Panlp, al so appears to regulate endocytosis (Wendland et al. 1996). Implication of eps15 in this pathway derived from the discovery of its constitutive association with the clathrin adaptor protein complex AP-2 (Benmerah et al. 1995). The related Eps15R protein is also associated in vivo to AP-2 (Iannolo et al. 1997; A. Salcini, P.G. Pelicci, and P. diFiore, unpubl.). Further characterization revealed that the carboxyl termini of Eps15 and Eps15R and the $\alpha$-subunit of AP-2 are involved in the interaction (Benmerah et al. 1996; Iannolo et al. 1997). In addition, immunomorphological analysis demostrated colocal ization of Eps15 and Eps15R with markers of the plasma membrane clathrincoated pits and vesicles (Tebar et al. 1996; A. Salcini, P.G. Pelicci, and P. diFiore, unpubl.).

Our present results might provide a molecular basis for the understanding of the protein-protein interactions involved in receptor-mediated internal ization, especially if viewed in the light of recent findings by $T$ an et al. (1996). This group demonstrated that the sequence N PFXD constitutes an internalization signal in Saccharomyces cerevisiae. In particular, they showed that N PFbased sequences in a Ste2p/Kex2p chimera and in Ste3p, the receptor for the amating factor ( $\mathrm{N}$ akayama et al. 


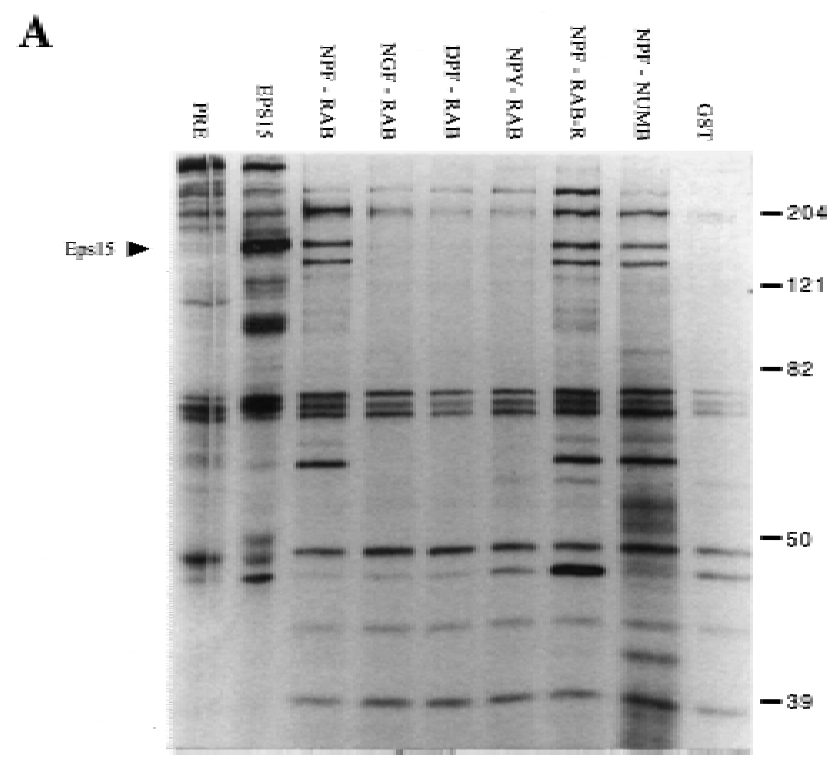

B

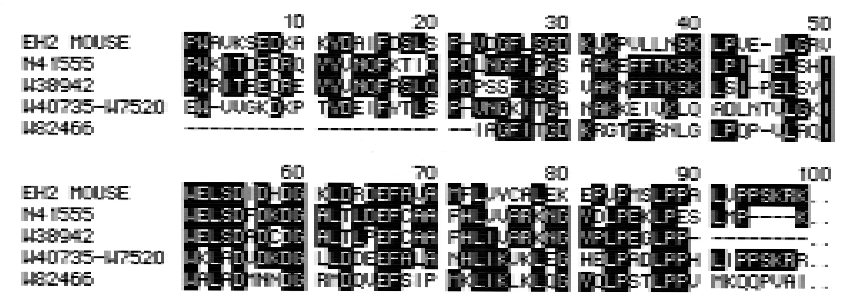

Figure 8. Identification of a family of $\mathrm{EH}$-containing proteins in mammals. (A) ${ }^{35} \mathrm{~S}$-Labeled lysates from N IH-3T $3\left(50 \times 10^{6}\right.$ TCAprecipitable counts) were incubated with the indicated GST fusion proteins displaying NPF-containing peptides (NPF-RAB, NPFRAB-R, and NPF-NUMB, underlined in Fig. 1C), mutant peptides (N GR-RAB, DPF-RAB, and NPY-RAB, shown in Fig. 6A), or with GST. The first two lanes represent the same lysate immunoprecipitated with anti-eps15 antibody (EPS15) or with a control serum (PRE). Specifically bound proteins were fractionated by SDS-PAGE and visualized by autoradiography. The position of Eps15 is indicated. Molecular mass markers are indicated in kD. (B) Identification of four novel EH-containing mammalian sequences. The EST db was screened with the TBLASTN al gorithm, using the three EH domains of Eps15 as a query. Only sequences displaying a $\mathrm{P}>10^{-5}$ were considered for further analysis, and they are indicated with their database accession numbers. The alignment of these sequences to the second EH domain of Eps15 is shown, as obtained by a CLUSTAL4 algorithm modified by visual inspection. The W40735-W7520 sequence derives from a merging of two shorter EST sequences of the same gene.

1985), are essential for endocytosis (Tan et al. 1996). Thus, the possibility that receptor-mediated endocytosis is regulated by EH-N PF interactions appears to be more than just a hypothesis.

The identification of an EH-mediated network of interactions sheds additional light on the function of the $\mathrm{EH}$ domain, in that EH-containing and EH-binding proteins are involved in more functions than internalization. Panlp, for instance, is required in yeast to maintain proper organization of the actin cytoskel eton (Tang and Cai 1996). NUMB, identified in this study as an EHbinding protein, is involved in cell fate determination through asymmetrical distribution as mitosis (Uemura et al. 1989; Rhyu et al. 1994; Spana et al. 1995), a function that requires precise sorting within the dividing cell. Thus, the EH-based network appears to be involved in processes connected with sorting and organization of molecules within the cell.

This contention is further strengthened by the identification of additional candidate EH-binding proteins. We searched databases for proteins containing multiple NPFs, as a characteristic of many of the EH-binding proteins identified in this study is the presence of multiple N PF-containing binding motifs, a feature that mirrors the frequent presence of $\mathrm{EH}$ domains in multiple copies. This search yielded proteins such as synaptojanin (three NPFs), which participates in synaptic vesicle recycling (M cPherson et al.1996); SCAM P 37 (three N PFs), which is part of a family of molecules that are components of membranes functioning in cell surface recycling (Brand and Castle 1993); and the Drosophila Dorsal protein (three N PFs), which establi shes a nucl ear vemtral-to-dor- sal gradient that is altered or absent in dorsalized or ventralized embryos, thus probably determining cell fate al ong the dorsal-ventral axis (Steward 1987). Other proteins containing multiple N PFs include the Drosophila Suppressor of sable protein (Voelker et al. 1991), the mammalian mitogen-responsive proteins p97, p93, and p67 (Xu et al. 1995), and the Stn-b protein of the Drosophila Stoned locus (GenBank accession no. U 54982). Although the EH-binding properties of these proteins will have to be validated experimentally, the hypothesis that the EH network is implicated in processes connected with transport, protein sorting, and organization of subcellular structures clearly appears worthy of further investigation.

Binding of Eps15 to NPF-containing targets did not appear to require tyrosine phosphorylation of the former, in that it was readily observed in in vitro bindings performed on serum-starved cell lysates, in which Eps15 is not phosphorylated. In addition, stoichiometry of binding was not improved under conditions in which a sizable fraction of Eps15 was tyrosine phosphorylated, as achieved in lysates from epidermal growth factor (EGF)stimulated cells (data not shown). N one of the Eps15binding abilities known so far, including binding to Crk and AP-2, appears to be phosphotyrosinedependent (Benmerah et al. 1995, 1996; Schumacher et al. 1995; lannolo et al. 1997). This raises the question of how the functional coupling of Eps15 to receptor tyrosine kinase (RTK)-activated pathways (Fazioli et al 1993; Wong et al. 1994) influences its downstream function(s), with particular regard to its $\mathrm{EH}$-mediated interactions. One attractive possibility is that a constitutive Eps15/target 
complex, such as Eps15/NUMB or Eps15/RAB, can be directed to a proper subcellular location by tyrosine phosphorylation. In this framework, tyrosine phosphorylation would act as a molecular switch through which RTKs can condition the targeting of components of the $\mathrm{EH}$ network to their active site within the cell.

\section{Materials and methods}

Screening of phage-display libraries

The peptide library utilized and the panning conditions were essentially as described by Felici et al. (1991), the main difference being that the target $\mathrm{EH}$ domains $(3 \mu \mathrm{g})$ were immobilized by binding to $20 \mu \mathrm{l}$ of GST-Sepharose matrix (Pharmacia). The peptide library was screened with either the GST-EH or the GST-EHR fusion protein, designed to encompass amino acid positions 2-330 and 15-368 of mouse Eps15 and Eps15R, respectively. Two panning cycles were carried out for each domain before the sequencing of the clones, which were enriched during the selection procedure.

\section{Isolation of cDNAs encoding EH-binding proteins}

A GST fusion protein containing the three EH domains of Eps15 (GST-EH; Wong et al. 1995) was used to screen a pCEV-LACbased prokaryotic expression library (a kind gift of T. Miki, National Institutes of Health, Bethesda, MD) from M 426 human fibroblasts. Recombinant plaques $\left(5 \times 10^{6}\right)$ were screened, after induction with IPTG, using a modification for the Far Western assay developed to identify proteins interacting with GST fusions (M atoskova et al. 1996a,b). Briefly, filters were blocked in $2 \%$ bovine serum albumin (BSA) in TTBS $(20 \mathrm{~mm}$ Tris- $\mathrm{HCl}$ at $\mathrm{pH} 7.5,150 \mathrm{~mm} \mathrm{~N} \mathrm{aCl}, 0.05 \%$ T ween 20) for at least $2 \mathrm{hr}$ at room temperature and then in reduced glutathione (Sigma, $3 \mu \mathrm{m}$ ) in TTBS with $0.5 \%$ (wt/vol) BSA for $1 \mathrm{hr}$ at room temperature. This latter step substantially reduced background (not shown). Blots were then incubated with GST-EH (10 nM) in TTBS in the presence of reduced glutathione $(3 \mu \mathrm{m})$ and BSA $(0.5 \% \mathrm{wt} / \mathrm{vol})$ for $1 \mathrm{hr}$ at room temperature. After extensive washing in TTBS, blots were detected with an affinity-purified anti-GST antibody, as described (M atoskova et al. 1996a,b).

Positive phages that specifically reacted with GST-EH but not with control GST were subjected to cross-hybridization experiments and assigned to seven groups, corresponding to distinct CDN As (not shown). For each group, phages containing the longest inserts were sequenced by the dideoxy-termination method on both strands of the CDNAs, using a commercial kit (Sequenase).

To obtain CDN A clones containing the entire ORFs of human NUMB, NU MB-R, RAB, and RAB-R, a pCEV-29-based eukaryotic expression library from M 426 human fibroblasts (a kind gift of T. Miki) were screened using standard techniques. Screening of the GenBank, EMBL, and EST databases was performed by the BLAST program (Altschul et al. 1990). Alignments of peptide sequences were performed by a CLUSTAL4 algorithm on MacDNASIS software.

\section{Production of recombinant proteins}

GST fusion proteins containing large fragments of the proteins of interest were obtained by recombinant PCR of the appropriate fragment from the murine Eps15 and Eps15R cDNAs or from the cDNAs of the EH-binding proteins, followed by clon- ing in the pGEX expression vector, in-frame with the GST moiety.

GST fusions containing short peptides from the proteins of interest were obtained by annealing in vitro complementary oligonucleotides with the appropriate sequence followed by cloning in-frame with the GST moiety in a gGEX-KT vector. Sequences of the oligonucleotides and primers used are available on request. Purification of the GST fusion protein onto agarose-glutathione and in vitro binding experiments were performed as described (Wong et al. 1995; Matoskova et al. 1996a,b).

\section{Protein studies}

Expression vectors for $\mathrm{HA}$-tagged RAB and NUMB proteins were engineered in the PMT2 eukaryotic expression vector by inserting (by insertional overlapping PCR) the sequence encoding the HA epitope (YDVPDYASLP) between codons 1 and 2 of the ORF of the RAB and NUMB CDNAs to obtain pMT2-HARAB and pMT2-HA-NUMB, respectively. The HA-NUMB/ NAA mutant was generated by changing the sequence coding for the unique NPF motif to a sequence coding for NAA by PCR-based oligonucleotide-directed mutagenesis ( $\mathrm{Ho}$ et al. 1989). Transient transfection of C33A cells by calcium phosphate was performed as described previously for $\mathrm{N} \mathrm{IH-3T} 3$ cells (Fazioli et al. 1993). Immunoprecipitation and immunoblotting experiments were performed as described previously (Fazioli et al. 1993; M atoskova et al. 1996a). Typically, we employed 50$100 \mu \mathrm{g}$ of total cellular proteins for direct immunoblot analysis and 3-5 mg of total cellular proteins for immunoprecipitation/ immunoblotting experiments. For coimmunopreciptiation experiments total cellular proteins were obtained in mild lysis conditions, in the absence of ionic detergents, to preserve protein-protein interactions, as described (Fazioli et al. 1993).

[35S]methionine-label ed RAB protein, employed in the experiments in Figure 7, was synthesized by in vitro transcriptiontranslation using a commercial kit (Promega) and the full-length RAB CDNA. M etabolic labeling with ${ }^{35}$ S]methionine of N IH3T 3 cells was performed as described previously (Fazioli et al. 1993).

\section{Acknowledgments}

This work was supported in part by grants from the Associazione Italiana Ricerca sul Cancro to P.P.D.F., P.G.P, and G.C. and from the Consiglio $\mathrm{N}$ azionale delle Ricerche to P.P.D.F. and P.G.P. The support of the European Community (BIOMED-2 Programme) to P.P.D.F. is also acknowledged. A.E.S. and E.S. are recipients of fellowships from the Fondazione Italiana Ricerca sul Cancro. M.D. is the recipient of a fellowship from the Fondazione Vollaro.

The publication costs of this article were defrayed in part by payment of page charges. This article must therefore be hereby marked "advertisement" in accordance with 18 USC section 1734 solely to indicate this fact.

\section{References}

Alschul, S.F., W. Gish, W. Miller, E.W. Myers, and D.J. Lipman. 1990. Basic local alignment search tool. J. Mol. Biol. 215: 403-410.

Benedetti, H., S. Raths, F. Crausaz, and H. Riezman. 1994. The END3 gene encodes a protein that is required for the internalization step of endocytosis and for actin cytoskel eton organization in yeast. Mol. Biol. Cell 5: 1023-1037. 
Benmerah, A., J. Gagnon, B. Begue, B. Megarbane, A. DautryVarsat, and N. Cerf-Bensussan. 1995. The tyrosine kinase substrate eps15 is constitutively associated with the plasma membrane adaptor AP-2. J. Cell Biol. 131: 1831-1838.

Benmerah, A., B. Begue, A. Dautry-Varsat, and N. Cerf-Bensussan. 1996. The ear of $\alpha$-Adaptin interacts with the $\mathrm{COOH}$ terminal domain of the Eps15 protein. J. Biol. Chem. 271: 12111-12116.

Blaikie, P., D. Immanuel, J. Wu, N. Li, V. Yainik, and B. Margolis. 1994. A region in shc distinct from the $\mathrm{SH} 2$ domain can bind tyrosine-phosphorylated growth factor receptors. J. Biol. Chem. 269: 32031-32034.

Bogerd, H.P., R.A. Fridell, S. Madore, and B.R. Cullen. 1995. Identification of a novel cellular cofactor for the Rev/Rex class of retroviral regulatory proteins. Cell 82: 485-494.

Brand, S.H. and J.D. Castle. 1993. SCAMP 37, a new marker within the general cell surface recycling system. EMBO J. 12: 3753-3761.

Burkholder, A.C. and L.H. Hartwell. 1985. The yeast al pha-factor receptor: Structural properties deduced from the sequence of the STE2 gene. Nucleic Acids Res. 13: 8463-8475.

Cicchetti, P., B.J. Mayer, G. Thiel, and D. Baltimore. 1992. Identification of a protein that binds to the $\mathrm{SH} 3$ region of $\mathrm{Abl}$ and is similar to Bcr and GAP-rho. Science 257: 803-806.

Cohen, G.B., R. Ren, and D. Baltimore. 1995. Modular binding domains in signal transduction proteins. Cell 80: 237-248.

Fazioli, F., L. Minichiello, B. Matoskova, W.T. Wong, and P.P. Di Fiore. 1993. Eps15, a novel tyrosine kinase substrate, exhibits transforming activity. Mol. Cell. Biol. 13: 5814-5828.

Felici, F., L. Castagnoli, A. Musacchio, R. Jappelli, and G. Ce sareni. 1991. Selection of antibody ligands from a large library of oligopeptides expressed on a multival ent exposition vector. J. Mol. Biol. 222: 301-310.

Feng, S., J.K. Chen, H. Yu, J.A. Simon, and S.L. Schreiber. 1994. Two binding orientations for peptides to the $\mathrm{SrC} \mathrm{SH} 3$ domain: Devel opment of a general model for SH3-ligand interactions. Science 266: 1241-1247.

Fritz, C.C., M.L. Zapp, and M.R. Green. 1995. A human nucleoporin-like protein that specifically interacts with HIV Rev. Nature 376: 530-533.

Gout, I., R. Dhand, I.D. Hiles, M.J. Fry, G. Panayotou, P. Das, O. Truong, N.F. Totty, J. Hsuan, and G.W. Booker. 1993. The GTPase dynamin binds to and is activated by a subset of $\mathrm{SH} 3$ domains. Cell 75: 25-36.

Herskovits, J.S., H.S. Shpetner, C.C. Burgess, and R.B. Vallee. 1993. Microtubules and Src homology 3 domains stimulate the dynamin GTPase via its C-terminal domain. Proc. Natl. Acad. Sci. 90: 11468-11472.

Ho, S.N., H.D. Hunt, R.M. Horton, J.K. Pullen, and L.R. Pease. 1989. Site-directed mutagenesis by overlap extension using the polymerase chain reaction. Gene 77: 51-59.

Iannolo, G., A.E. Salcini, I. Gaidarov, O.B. Goodman Jr., P.G. Pelicci, P.P. Di Fiore, and J.H. Keen. 1997. Mapping of the molecular determinants involved in the interaction between eps15 and AP-2. Cancer Res. 57: 240-245.

Kavanaugh, W.M. and L.T. Williams. 1994. An alternative to $\mathrm{SH} 2$ domains for binding tyrosine phosphorylated proteins. Science 266: 1862-1865.

Matoskova, B., W.T. Wong, N. Seki, T. Nagase, N. N omura, K.C. Robbins, and P.P. Di Fiore. 1996a. RN-tre identifies a family of tre-related proteins displaying a novel potential protein binding domain. O ncogene 12: 2563-2571.

Matoskova, B., W.T. Wong, N. N omura, K.C. Robbins, and P.P. Di Fiore. 1996b. RN -tre specifically binds to the SH 3 domain of eps8 with high affinity and confers growth advantage to NIH3T3 upon carboxy-terminal truncation. Oncogene
12: 2679-2688.

McPherson, P.S., E.P. Garcia, V.I. Slepnev, C. David, X. Zhang, D. Grabs, W.S. Sossin, R. Bauerfeind, Y. Nemoto, and P. De Camilli. 1996. A presynaptic inositol-phosphatase. Nature 379: 353-357.

Musacchio, A., M. Wilmanns, and M. Saraste. 1994. Structure and function of the SH3 domain. Prog. Biophys. Mol. Biol. 61: 283-297.

N akayama, N ., A. Miyajima, and K. Arai. 1985. Nucleotide sequences of STE2 and STE3, cell type-specific sterile genes from Saccharomyces cerevisiae. EMBO J. 4: 2643-2648.

Pleiman, C.M., W.M. Hertz, and J.C. Cambier. 1994. Activation of phosphatidylinositol-3' kinase by Src-family kinase $\mathrm{SH} 3$ binding to the p85 subunit. Science 263: 1609-1612.

Ren, R., B.J. Mayer, P. Cicchetti, and D. Baltimore. 1993. Identification of a ten-amino acid proline-rich $\mathrm{SH} 3$ binding site. Science 259: 1157-1161.

Rhyu, M.S., L.Y. Jan, and Y.N. Jan. 1994. Asymmetric distribution of numb protein during division of the sensory organ precursor cell confers distinct fates to daughter cells. Cell 76: 477-491.

Schlessinger, J. 1994. SH2/SH3 signaling proteins. Curr. O pin. Genet. Dev. 4: 25-30.

Schumacher, C., B.S. Knudsen, T. Ohuchi, P.P. Di Fiore, R.H. Glassman, and $\mathrm{H}$. Hanafusa. 1995. The SH3 domain of Crk binds specifically to a conserved prolinerich motif in Eps15 and Eps15R. J. Biol. Chem. 270: 15341-15347.

Songyang, Z., S.E. Shoel son, M. Chaudhuri, G. Gish, T. Pawson, W.G. Haser, F. King, T. Roberts, S. Ratnofsky, and R.J. Lechleider. 1993. SH2 domains recognize specific phosphopeptide sequences. Cell 72: 767-778.

Songyang, Z., S.E. Shoelson, J. McGlade, P. Oliver, T. Pawson, X.R. Bustelo, M. Barbacid, H. Sabe, H. Hanafusa, and T. Yi. 1994. Specific motifs recognized by the SH 2 domains of Csk, 3BP2, fps/fes, GRB-2, HCP, SHC, Syk, and Vav. Mol. Cell. Biol. 14: 2777-2785.

Spana, E.P., C. Kopczynski, C.S. Goodman, and C.Q. Doe. 1995. Asymmetric localization of numb autonomously determines sibling neuron identity in the Drosophila CNS. Development 121: 3489-3494.

Sparks, A., J. Rider, N. Hoffman, D. Fowlkes, L. Quilliam, and B. Kay. 1996. Distinct ligand preferences of Src homology 3 domains from Src, Y es, A bl, Cortactin, p53bp2, PLC gamma, Crk, and Grb2. Proc. Natl. Acad. Sci. 93: 1540-1544.

Steward, R. 1987. Dorsal, an embryonic polarity gene in Drosophila, is homologous to the vertebrate proto-oncogene, crel. Science 238: 692-694.

Tan, P.K., J.P. Howard, and G.S. Payne. 1996. The sequence NPFXD defines a new class of endocytosis signal in Saccharomyces cerevisae. J. Cell. Biol. 135: 1789-1800.

Tang, H.-Y. and M. Cai. 1996. The EH-containing protein Pan1 is required for normal organization of the actin cytoskel eton in Saccaromyces cerevisiae. Mol. Cell. Biol. 16: 4897-4914.

Tebar, F., T. Sorkina, A. Sorkin, M. Ericsson, and T. Kirchhausen. 1996. Eps15 is a component of clathrin-coated pits and vesicles and is located at the rim of coated pits. J. Biol. Chem. 271: 28727-28730.

U emura, T., S. Shepard, L. Ackerman, L.Y. Jan, and Y.N. Jan. 1989. Numb, a gene required in determination of cell fate during sensory organ formation in Drosophila embryos. Cell 58: 349-360.

van der Geer, P. and T. Pawson. 1995. The PTB domain: A new protein module implicated in signal transduction. Trends Biochem. Sci. 20: 277-280.

Verdi, J.M., R. Schmandt, A. Bashirullah, S. Jacob, R. Salvino, C.G. Craig, A.E. Program, H.D. Lipshitz, and C.J. McGlade. 
1996. M ammalian N UM B is an evolutionarily conserved signaling adapter protein that specifies cell fate. Curr. Biol. 6: 1134-1145.

Voelker, R.A., W. Gibson, J.P. Graves, J.F. Sterling, and M.T. Eisenberg. 1991. The Drosophila suppressor of sable gene encodes a polypeptide with regions similar to those of RN Abinding proteins. Mol. Cell. Biol. 11: 894-905.

Wendland, B., J.M. M cCaffery, Q. Xiao, and S.D. Emr. 1996. A novel fluorescence-activated cell sorter-based screen for yeast endocytosis mutants identifies a yeast homologue of mammalian eps15. J. Cell. Biol. 135: 1485-1500.

Wong, W.T., M.H. Kraus, F. Carlomagno, A. Zelano, T. Druck, C.M. Croce, K. Huebner, and P.P. Di Fiore. 1994. The human eps15 gene, encoding a tyrosine kinase substrate, is conserved in evolution and maps to 1p31-p32. Oncogene 9: 1591-1597.

Wong, W.T., C. Schumacher, A.E. Sal cini, A. Romano, P. Castagnino, P.G. Pelicci, and P.P. Di Fiore. 1995. A protein-binding domain, $\mathrm{EH}$, identified in the receptor tyrosine kinase substrate Eps15 and conserved in evolution. Proc. Natl. Acad. Sci. 92: 9530-9534.

Yu, H., J.K. Chen, S. Feng, D.C. Dal garno, A.W. Brauer, and S.L. Schreiber. 1994. Structural basis for the binding of prolinerich peptides to SH 3 domains. Cell 76: 933-945.

Xu, X.X., W. Yang, S. Jackowski, and C.O. Rock. 1995. Cloning of a novel phosphoprotein regulated by colony-stimulating factor 1 shares a domain with the Drosophila disabled gene product. J. Biol. Chem. 270: 14184-14191.

Zhong, W., J.N. Feder, M.M. Jang, L.Y. Jan, and Y.N. Jan. 1996. Asymmetric localization of a mammalian numb homolog during mouse cortical neurogenesis. Neuron 17: 43-53.

Zhou, M-M., K.S. Ravichandran, E.T. Olejniczak, A.M. Petros, R.P. M eadows, M. Sattler, J.E. Harlan, W.S. Wade, S.J. Burakoff, and S.W. Fesik. 1995. Structure and ligand recognition of the phosphotyrosine binding domain of shc. Nature 378: 584-592. 


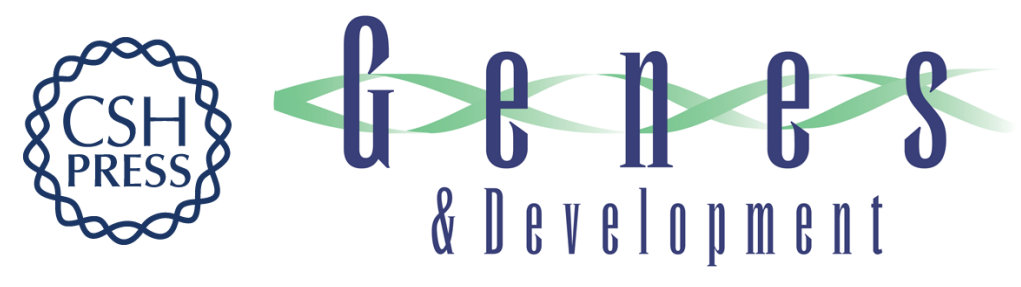

\section{Binding specificity and in vivo targets of the EH domain, a novel protein-protein interaction module}

Anna Elisabetta Salcini, Stefano Confalonieri, Margherita Doria, et al.

Genes Dev. 1997, 11:

Access the most recent version at doi:10.1101/gad.11.17.2239

References

This article cites 48 articles, 24 of which can be accessed free at: http://genesdev.cshlp.org/content/11/17/2239.full.html\#ref-list-1

License

Email Alerting

Receive free email alerts when new articles cite this article - sign up in the box at the top Service right corner of the article or click here.

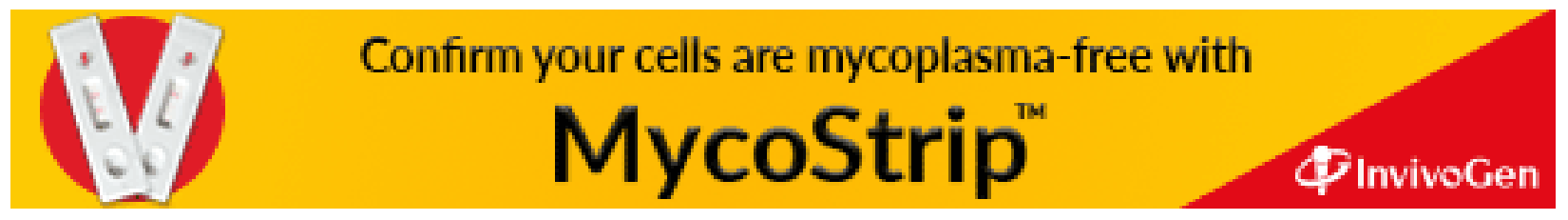

